expenditure, also experienced more adverse trends in premature mortality.

Methods We carried out a longitudinal ecological analysis using data on housing services net expenditure in 146 upper tier local authorities in England between 2013 and 2018, linked to all-cause premature mortality rate (deaths under 75 years) for males and females. To analyse local authority expenditure on housing services we utilised Revenue Outturn data provided by the Ministry of Housing, Communities \& Local Government; data on premature mortality was acquired from Public Health England. We used an instrumental variable approach to investigate this relationship to address model endogeneity. We used central government funding allocated to local authorities as an instrument because we expect it to influence health through its impact on levels of service expenditure but not influence health outcomes directly. We analysed the relationship between housing services spending and mortality using two-stage least squares linear regression with robust clustered standard errors and fixed area and time effects. We also adjusted for time-varying confounding effects of local economic conditions. We calculated our models with alternative specifications to test the robustness of our findings.

Results Average expenditure per person on housing services decreased from $£ 41$ in 2013 to $£ 30$ in 2018 . Each $£ 10$ per person reduction was associated with a 17.6 increase in premature mortality rate for males (95\% CI: 2.1 to 33.0 ) and 12.6 in females (95\% CI: 2.1 to 23.1 ). Over the sixyear period, reductions in spending were associated with 8,900 additional premature deaths $(95 \%$ CI: 1,200 to 16,500).

Conclusion Reduction in spending for housing services may in part explain recent adverse trends in mortality in England. Investment in housing and homelessness support is likely to have a positive impact on health outcomes. Limitations of this study include restricting the time period of analysis to after 2013 due to changes in local government funding policy, and the reliance on area-level mortality data calculated over 3-year periods.

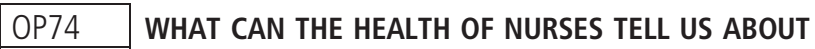 INEQUALITIES?}

William Ball*. Centre for Health Data Science, University of Aberdeen, Aberdeen, UK

10.1136/jech-2021-SSMabstracts.74

Background Unfair and avoidable inequalities in health, observed for decades, are pervasive and persistent in the United Kingdom and beyond. Policies that aim to reduce inequalities, or at least improve population health in the last decade have focused disproportionately on individual or behavioural factors, with little positive effect. Studying the health of a socioeconomically homogenous sample of Nurses, with intuitively preferential individual characteristics like high levels of education, can serve as a counterfactual - what would our health, and inequalities by deprivation, look like if we all had similar characteristics?

Methods Individual-level records $(\mathrm{n}=478,802)$ from the nationally representative ONS (England \& Wales) and Scottish Longitudinal Studies, linked to an adjusted UK-comparable measure of small-area deprivation have been used to compare self-rated health and inequalities in samples of economically active Nurses and Non-Nurses. Descriptive and correlational statistics have been used to assess the relative homogeneity of Nurses to Non-Nurses as well as wider trends in self-rated health and inequalities based on small-area deprivation. In addition, a logistic regression model was built to estimate the effect of Nurses status on self-rated health whilst adjusting for area deprivation and other potential confounders.

Results Nurses are older, predominantly female and are more socioeconomically homogenous than Non-Nurses measured on individual characteristics such as occupational social class or highest level of education. Nurses are more likely than NonNurses to live in the least deprived areas (45\% vs. $41 \%)$ and report Very Good Self-Rated Health (59\% vs. 52\%). A social gradient by area deprivation exists for those reporting less than good health in both Nurses (Least deprived - 8\%, Most deprived - 10\%) and Non-Nurses (Least - 9.9\%, Most 18.4\%). However, at each level of deprivation Nurses are less likely to report less than good health than Non-Nurses. A logistic regression model, adjusting for demographic characteristics \& area deprivation found that the odds of reporting good or better health for Nurses was 1.33 (SLS - 95\% CI 1.19 - 1.49) and 1.41 (ONS LS - 95\% CI 1.32 - 1.52) times that of Non-Nurses.

Conclusion Nurses report better self-rated health than NonNurses and this persists even after adjustment for socioeconomic and demographic differences. This finding is consistent with analysis showing preferential health behaviours in UK Nurses. However, a social gradient by area deprivation still exists, even for a population with preferential individual socioeconomic characteristics.

\section{OP75 MAPPING UK POLICIES AND STRATEGIES RELEVANT TO CHILD AND MATERNAL HEALTH TO IDENTIFY OPPORTUNITIES FOR UPSTREAM EVALUATIONS: INITIAL FINDINGS FROM THE MATERNAL AND CHILD HEALTH NETWORK (MATCHNET)}

${ }^{1}$ Emma Stewart*, ${ }^{1}$ Anna Pearce, ${ }^{2}$ Joanne Given, ${ }^{3}$ Ruth Gilbert, ${ }^{4}$ Sinead Brophy, ${ }^{5}$ Richard Cookson, ${ }^{3}$ Pia Hardelid, ${ }^{6}$ Katie Harron, ${ }^{1}$ Alastair Leyland, ${ }^{7}$ Rachael Wood. ${ }^{7} \mathrm{MRC} /$ CSO Social and Public Health Sciences Unit, University of Glasgow, Glasgow, UK; ${ }^{2}$ Institute of Nursing and Health Research, UIster University, Belfast, UK; ${ }^{3}$ NIHR Children and Families Policy Research Unit, UCL Great Ormond Street Institute of Child Health, London, UK; ${ }^{4}$ School of Medicine, Swansea University, Swansea, UK; ${ }^{5}$ Centre for Health Economics, University of York, York, UK; ${ }^{6}$ Great Ormond Street Institute of Child Health, UCL, London, UK; ${ }^{7}$ Clinical and Public Health Intelligence Team, Public Health Scotland, Edinburgh, UK

\subsection{6/jech-2021-SSMabstracts.75}

Background Interventions to tackle the social determinants of health can improve outcomes during pregnancy and early childhood, leading to better health across the life-course. Variation in content, timing, and implementation of policies across the 4 UK nations holds great potential for quasiexperimental evaluations. We aimed to adapt systematic review methods to identify UK policies that potentially affect maternal and child health across the social determinants of health framework; and determine suitable candidates for quasi-experimental evaluation using administrative data.

Methods A systematic search strategy comprised open keyword (i.e. 'child', 'child health', 'child and maternal health') and category searches of UK Government websites (e.g. Children and Families, Education, Health and Social Care, Welfare) and extensive hand searching of existing policy reviews until 